\title{
Quality Control and Evaluation Methods of Concrete Engineering and Its Reliability Analysis
}

\author{
Xiaojian Wang* and Kaiyu Jiang
}

Chongqing Three Gorges University, Chongqing, China

\begin{abstract}
Ultrasonic pulse velocity (UPV) and rebound hammer (RH) tests are often used for assessing the quality of concrete and estimation of its compressive strength. Several parameters influence the property of concrete, such as the type and size of aggregates, cement content and the implementation of concrete. To account for these factors, both of the two tests are combined and their measurements are calibrated with the results of mechanical tests on cylindrical specimens cast on site. In this study, the two tests cited above have been used to determine the concrete quality by applying regression analysis models between compressive strength of in situ concrete on existing structure and the nondestructive tests values. With the combined method, equations are derived using statistical analysis (simple and multiple regression) to estimate compressive strength of concrete on site. Besides, this paper presents the reliability analysis to nonlinear reinforced concrete beams. A First Order Reliability Method (FORM) is used, and the results are compared to the ones given by Monte Carlo simulation.
\end{abstract}

Keywords: Concrete engineering, evaluation criteria, reliability analysis, quality control.

\section{INTRODUCTION}

Evaluation of concrete properties is of great interest, whether to detect altered areas or to control the concrete quality and estimate its compressive strength [1]. The standard methods used to assess the quality of concrete in concrete structures on specimens cannot be considered. The disadvantage is that results are not immediately known, and the number of specimens or samples is insufficient for an economic reason. Besides, it does not reflect the reality of the structure [2].

The main advantage of nondestructive testing method is to avoid the concrete damage on the performance of building structural components. Additionally, their usage is simple and quick [3]. Test results are available on site. Concrete testing in structures is demanding in which the cores cannot be drilled, where the use of less expensive equipment is required.

Several nondestructive evaluation methods have been developed. These methods are almost based on the fact that some physical properties of concrete can be related to the compressive strength of concrete. The Schmidt rebound hammer (SRH) [4] and the ultrasonic pulse velocity (UPV) tests [5], are combined to develop correlation between hammer/ultrasonic pulse velocity readings and the compressive strength of the concrete. These nondestructive measurements have been proven to be an effective tool for inspection of concrete quality.

Reinforced concrete structures present significant nonlinear behavior and consequently nonlinear analysis of this kind of structure has been subject of research for many years [6]. In the last decades very accurate structural models were proposed, that can take into account most aspects of the nonlinear behavior of concrete structures. However, reinforced concrete structures (as is the case of most structures) are subjected to strong uncertainties, both related to the properties of the material and the applied loads [7]. Consequently, the design of structures that will need to work under real conditions need to take into account these uncertainties to some degree. Until about 1960 these uncertainties were considered by applying some safety factor during the design stage. However, these safety factors were established only by means of "engineering judgment", and not by a rigorous scientific approach.

The next step was the design of structures according to limit states, that is the approach recommended by most structural design standards nowadays. In this case, the properties of each material and the magnitude of each load is decreased/increased according to its respective factor [8]. These factors are evaluated based on probabilistic analysis and presented as fixed values in design standards. For this reason design using limit states is also known as semiprobabilistic design. The factors were actually evaluated using probabilistic analysis, but the designer makes a deterministic analysis using reduced/increased resistances/loads.

It turns out that design standards are not able to cover the full range of application that engineers are able to conceive [9]. Even if some kind of design standards are available for most kind of constructions (such as buildings, bridges and dams), sometimes the engineers need to design some structure that does not fit exactly in any standard due to its size, complexity or multidisciplinary nature. In these cases (or in cases that the engineer wants to) probabilistic analysis can be pursued. Full probabilistic analysis, where one aims for a full 
probabilistic characterization of the behavior of the structure, needs in general much computational effort. Fortunately, in many cases it is enough to study the structure from the optics of "fail" versus "do not fail". In these cases one can substitute a full probabilistic analysis by a reliability analysis, where only the failure probability is evaluated. This takes much less computational effort, and can be successfully applied to several structural problems.

In this paper we first present evaluation methods of concrete engineering, and then propose an approach for the reliability analysis of reinforced concrete beams and frames using a First Order Reliability Method (FORM). Failure is assumed to occur when the displacements are bigger than some prescribed limit. Besides, the FORM algorithm is applied directly to the problem, and thus an efficient approach for carrying out sensitivity analysis is also presented. It is also proven that the reliability index given by this problem is a lower bound for the reliability index when collapse of the structure is considered. Finally, two examples are presented in order to validate the proposed approach.

\section{EVALUATION OF CONCRETE STRUCTURES BY NONDESTRUCTIVE METHODS}

Nondestructive testing (NDT) methods are test methods that rely upon physical or chemical principles that can be monitored or measured without significantly affecting the appearance or performance of the analyzed structures. For this reason, NDT methods are favored when testing many structural materials and structures. According to Nesvijski [3], they allow evaluation in situ during service and enable monitoring for an extended period of time.

\subsection{Ultrasonic Pulse Velocity}

Ultrasonic testing is a well-established nondestructive technique for the detection of flaws and characterization of materials, based on the monitoring of the propagation speed of an ultrasonic wave throughout the material. During tests, transmitting and receiving transducers are placed on different parts of the object, allowing an ultrasonic pulse to be sent through the material. If a flaw is encountered, the wave is delayed. Using this knowledge, UPV can be used as an assessment tool to help quantify the existence extent and progression of damage in different structures.

Although most applications of this test focus on the inspection of metals, ultrasonic pulse transmission technique has been shown to be useful also for testing concrete elements. UPV have already been applied to new and old structures, slabs, columns, walls, fire damaged areas, hydroelectric structures, pipes, prefab, and pre-stressed beams. The UPV method is particularly effective, powerful, and flexible, allowing in-depth analysis of material homogeneity. It is possible to determine concrete uniformity, to control its quality, to follow up the deterioration, to check the presence of internal flaws and voids using UPV, and to make comparisons with reference specimens, it may estimate potential compressive strength. Finally, when regularly used, it may provide data on the development of problems.

The evaluation of ultrasonic results is a highly specialized and complex activity, which requires careful data col- lection and expert knowledge and sensitivity to obtain reliable diagnosis. In order to map the homogeneity of a structure, it is necessary to interpret and connect a large number of UPV readings. Accurate analysis requires reliable interpretation strategies, but there is not yet a proper widely accepted method to analyze this data. The research related in this work aimed to investigate a strategy to analyze these data using 3D mapping techniques used to build topographic models.

\subsection{Rebound Hammer}

The rebound hammer test is essentially a surface hardness tester, where an elastic mass is projected against the surface and the rebound is measured, resulting in the $\mathrm{RH}$ number. The rebound mass depends on the hardness of the surface against which the mass impinges. Of course there is a relationship between rebound number and surface strength but deducing concrete strength from the RH number must be done with great care, because several superficial effects can affect the rebound without affecting concrete resistance. Despite the weak theoretical relationship between concrete strength and rebound number, several empirical correlations have been created and used, with mixed success.

The key to understand the inherent limitations of the test for estimating strength is recognizing the factors influencing rebound distance. Essentially, it is a complex problem of impact loading and stress-wave propagation. The rebound distance depends on the kinetic energy in the hammer before impact with the shoulder of the plunger and the amount of that energy absorbed during the impact. Part of the energy is absorbed as mechanical friction in the instrument, and part of the energy is absorbed in the interaction of the plunger with the concrete. It is the latter factor that makes the rebound number an indicator of the concrete properties. The energy absorbed by the concrete depends on the stress-strain relationship of the concrete. Nowadays, the RH method is mostly used to make a preliminary assessment of uniformity or to determine suspicious regions where low or damaged concrete may be located. Given the easiness of application, a quick map of large areas can be done in little time. That is the way the test was used in the present study.

\section{STATEMENT OF THE RELIABILITY PROBLEM}

\subsection{The Failure Function}

In order to simplify the numerical model and reduce the computational effort involved, we assume here an approximated piecewise linear stress-strain relation as the one shown in Fig. (1). In the reliability problem, failure is assumed to occur when the displacement of a given node of the structure is bigger than a prescribed limit. Thus, the failure function can be written as

$f(\boldsymbol{x}, q)=q_{j}(\boldsymbol{x})-q_{\max }$

where $q_{j}(\boldsymbol{x})$ is the displacement in a given node, $q_{\max }$ is the maximum allowable displacement for this node and $\boldsymbol{x}$ is the vector of probabilistic variables, named from now on as parameters of the reliability problem. From (1) it can be seen that failure occurs when $f>0$, that is, when the displacement is bigger than the allowable displacement. 
The FORM also uses the gradient of the failure function according to the parameters of the problem in order to evaluate the reliability of the structure. The gradient of (1) is

$\nabla_{x} f=\nabla_{x} q_{j}$

since $q_{\max }$ is a fixed value. Thus, in order to evaluate the gradient of the failure function it is necessary to evaluate the gradient of the displacements according to the parameters of the problem. The evaluation of this information is described later.

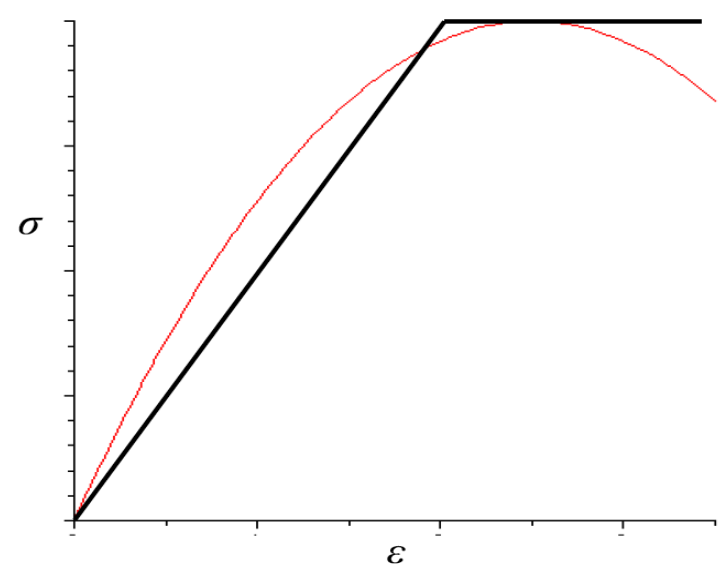

Fig. (1). the parabolic approximation (red) for the stress-strain relation for concrete under uniaxial compression and the piecewise linear approximation (black).

\subsection{The Relationship between the Problem Defined for Allowable Displacements and the Problem Defined for Collapse}

From the computational point of view, collapse of the structure happens when the tangent stiffness matrix becomes singular and thus any small load increment leads to very big displacements. That is, for a structure that collapses some displacements go to infinity. the reliability analysis problem for collapse of the structure can be defined as

$$
f(\boldsymbol{x}, q)=\lim _{q_{\max } \rightarrow \infty}\left\{q_{j}(\boldsymbol{x})-q_{\max }\right\}
$$

where $q_{j}(\boldsymbol{x})$ is the d.o.f. according to which collapse is defined. According to (3), the structure would be safe for a finite displacement $q_{j}(\boldsymbol{x})$ and unsafe when this displacement goes to infinity. For computational purposes (3) can be rewritten as

$$
f(\boldsymbol{x}, q)=q_{j}(\boldsymbol{x})-b
$$

where $b$ is taken as a sufficiently big number, in order to take the role of the limit that appears in (4). Note that in most practical cases the displacements are of the order of $10^{-6} \mathrm{~m}$ to $10^{-2} \mathrm{~m}$, and taking $b=10$ would likely do the job.

In practice, considering such a big allowable displacement would lead to serious convergence difficulties. That's because the FORM would eventually move to a point in the design space that leads to collapse of the structure. However, in such points the displacements would be very big and the evaluation of the failure function and its gradient would surely fail. From this step onward, the FORM would start to move almost randomly, since the information given by the failure function and its gradient would not represent the problem appropriately. Consequently, solving the reliability analysis problem considering collapse of the structure using an approach similar to the ones given by (3) and (4) is not an efficient approach. Fortunately, it is possible to evaluate a lower bound for the reliability index of this problem.

\section{SENSITIVITY ANALYSIS}

As discussed previously, FORM algorithms need to evaluate the gradient of the failure function according to the design variables in order to carry out the reliability analysis. The procedure involved in evaluating this information is known in literature as sensitivity analysis. Suppose that the structure being studied has $n$ degrees of freedom and that the sensitivity analysis is made for $\mathrm{m}$ design variables (parameters of the reliability problem). Besides, the design variables are all grouped in a vector $\boldsymbol{x}$. In this case, sensitivity analysis of the displacements is accomplished when the following matrix is evaluated:

$$
\nabla_{x} q=\left[\begin{array}{llll}
\frac{\partial q}{x_{1}} & \frac{\partial q}{x_{2}} & \ldots & \frac{\partial q}{x_{m}}
\end{array}\right]
$$

that is the gradient of the nodal displacements $q$ according to the design variables $\boldsymbol{x}$.

The gradient from (5) can be evaluated using some finite difference scheme, by applying small changes to each design variable and then solving the entire structural problem again. However, this approach implies solving the nonlinear structural problem several times, at least once for each design variable. Since the computational cost needed for a single nonlinear structural analysis is generally high (since several systems of linear equations must be solved for each analysis), evaluating (5) using finite differences can lead to almost prohibitive computational costs.

Another approach for evaluating (5) is by using some technique from sensitivity analysis. Some very efficient approaches are discussed by Haftka and Gürdal (1992), but here we use a slightly modified version of a technique that is generally applied to linear structural problems.

First, we differentiate (1) according to some design variable $x_{j}$ and rearrange to get

$$
\boldsymbol{M}_{s} \frac{\partial q}{\partial x_{j}}=\frac{\partial \boldsymbol{F}}{\partial x_{j}}-q \frac{\partial \boldsymbol{M}_{s}}{\partial x_{j}}
$$
by

The partial derivative of the stiffness matrix $\boldsymbol{M}_{s}$ is given

$$
\frac{\partial \boldsymbol{M}_{s}}{\partial x_{j}}=\frac{d \boldsymbol{M}_{s}}{d x_{j}}+\sum_{i=1}^{n} \frac{d \boldsymbol{M}_{s}}{d q_{i}} \frac{\partial q_{i}}{\partial x_{j}}+\sum_{i=1}^{n} \frac{d \boldsymbol{M}_{s}}{d F_{i}} \frac{\partial F_{i}}{\partial x_{j}}
$$

where $d$ stands for an ordinary derivative that does not take into account the implicit relation between $\boldsymbol{M}_{s}$ and $x_{j}$ by means of $q$ or $\boldsymbol{F}$.

According to (7), changes to a parameter $x_{j}$ can lead to changes to the stiffness matrix $\boldsymbol{M}$ by three different ways, namely by its direct influence on the stiffness matrix, by its influence on the displacements $q$ and by its influence on the loads $\boldsymbol{F}$. In general, the parameters $x_{j}$ will exert small or no 
influence at all on the applied loads, and thus the last term of (7) can be neglected. This is not true when the parameter $x_{j}$ is actually some applied load, but this case is discussed further on. Besides, it is expected that the indirect influence of $x$ to the stiffness matrix, by means of the displacements will be small if compared to its direct influence. Thus, the second term from (7) is also neglected. Consequently, the following approximation is made to (7):

$\frac{\partial \boldsymbol{M}_{s}}{\partial x_{j}} \approx \frac{d \boldsymbol{M}_{s}}{d x_{j}}$

Substituting (8) into (6) we get

$\boldsymbol{M}_{s} \frac{\partial q}{\partial x_{j}} \approx \frac{\partial \boldsymbol{F}}{\partial x_{j}}-q \frac{d \boldsymbol{M}_{s}}{d x_{j}}$

Sensitivity analysis is then made by solving (9) for the partial derivatives $\partial q / \partial x_{j}$. Since the system of linear equations are solved for the same secant stiffness matrix $\boldsymbol{M}_{s}$, decomposition procedures (such as Cholesky or L.U. decomposition) can be used efficiently. These techniques decompose the stiffness matrix into a product of two matrices that are lower triangular and upper triangular. Each system of linear equations involved in obtaining $\partial q / \partial x_{j}$ is then solved using retro substitution. This is a very efficient procedure when one needs to solve several systems of linear equations for the same coefficients matrix, since the main computational effort lies in the decomposition procedure itself, that is made only once.

\section{NUMERICAL RESULTS}

The first example is that from Fig. (2). The cross section has $h=30 \mathrm{~cm}$ and $b=20 \mathrm{~cm}$. The beam is $4 \mathrm{~m}$ long and there is an applied force at mid span. Three reinforcement bars of $12 \mathrm{~mm}$ are used in the upper chord and other three bars of $12 \mathrm{~mm}$ are used in the lower chord. The covering distance is $c=2.5 \mathrm{~cm}$ for both reinforcements. The material properties are taken as $\sigma_{v c}^{c}=25 \mathrm{MPa}, \sigma_{y t}^{c}=2.5 \mathrm{MPa}, \varepsilon_{v c}^{c}=2 / 1000, \varepsilon_{v t}^{c}=$ $0.2 / 1000, \varepsilon_{u c}^{c}=3 / 1000, \varepsilon_{u t}^{c}=0.3 / 1000, \sigma_{y}^{s c}=450 \mathrm{MPa}, \varepsilon_{y}^{s}=$ $2.25 / 1000$ and $\varepsilon_{u}^{s}=35 / 1000$. The beam is divided into 8 finite elements of equal length.
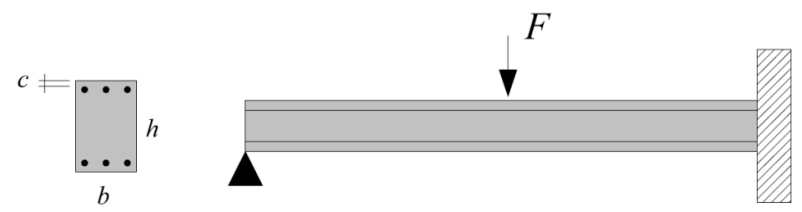

Fig. (2). Reinforced concrete beam used in the first example.

The displacement at mid span for different magnitudes of the applied load are presented in Fig. (3). It can be seen that the behavior of the model is consistent. For a load of about 2.5 tons the concrete is not able to resist tension anymore (it starts to crack), and so there is a change on the displacement evolution. For a load of about 8 tons the steel starts to yield finally leading to the collapse of the structure. This example was presented in order to show that even if the structural model is very simple it is able to capture the main nonlinear behavior of reinforced concrete structures.

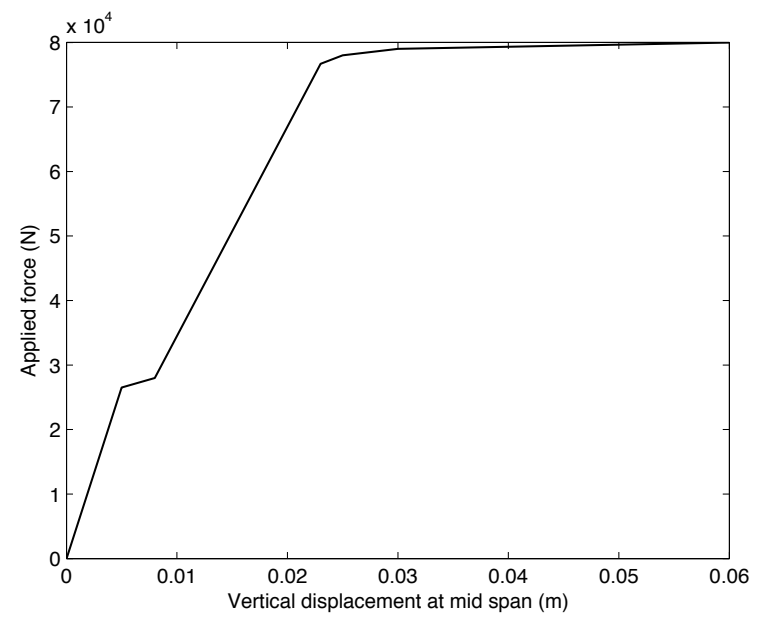

Fig. (3). Displacement $\boldsymbol{x}$ applied force for the beam used in the first example.

We now address the reliability analysis for this problem. The applied load is assumed to be a gaussian variable with mean equal to $5 \mathrm{E} 4 \mathrm{~N}$ (tons) and standard deviation equal to $0.5 \mathrm{E} 4 \mathrm{~N}$ (tons). This is called here parameter $x_{1}$. Besides, the standard deviation of the concrete yielding stress in compression $\sigma_{v c}^{c}$ is assumed to have an standard deviation of $2.5 \mathrm{MPa}$, while the standard deviation of the steel yielding stress $\sigma_{y}^{s}$ is assumed to have an standard deviation of $10 \mathrm{MPa}$. 'These variables are named here parameters $x_{2}$ and $x_{3}$, respectively. The other parameters are taken as deterministic variables with values as defined previously. The reliability analysis of this example is made assuming two allowable displacements at mid span, equal to $0.020 \mathrm{~m}$ (Case 1) and equal to $0.023 \mathrm{~m}$ (Case 2).

The results are presented in Tables $\mathbf{1}$ and $\mathbf{2}$. The reliability index for Case 1 is 3.4850, while for Case 2 it is 5.0796. As expected, the reliability index increases when a larger displacement is allowed. In order to study the influence of the probabilistic variables, we increase the standard deviation of the applied load to $1.0 \mathrm{E} 4 \mathrm{~N}$ (tons), the standard deviation of the concrete yielding stress in compression to 3.0MPa, and the standard deviation of the steel yielding stress to $20 \mathrm{MPa}$. The allowable displacement is taken as $0.020 \mathrm{~m}$. This example is called here Case 3 . The reliability index for this case is 1.7667 and the results are presented in Tabels 1 and 2. This results was also expected, since increasing the standard deviation of the random variables leads to a less reliable structure.

Table 1. Most probable failure point at the normalized space for the reliability analysis of the first example.

\begin{tabular}{|c|c|c|c|c|}
\hline Case & $x_{1}$ & $x_{2}$ & $x_{3}$ & $\beta$ \\
\hline \hline 1 & 3.4317 & -0.4387 & -0.4201 & 3.4850 \\
\hline 2 & 4.9815 & -7.1527 & -6.8900 & 5.0796 \\
\hline 3 & 1.7487 & -1.3104 & -2.1509 & 1.7667 \\
\hline
\end{tabular}


Table 2. Most probable failure point at the real space for the reliability analysis of the first example.

\begin{tabular}{|c|c|c|c|}
\hline Case & $\boldsymbol{F}(\mathbf{E} 4 \mathbf{N})$ & $\boldsymbol{f}_{\mathbf{c}}(\mathbf{M P a})$ & $\boldsymbol{f}_{\mathbf{s}}(\mathbf{M P a})$ \\
\hline \hline 1 & 6.7158 & 23.9033 & 445.7988 \\
\hline 2 & 7.4908 & 23.2118 & 443.1099 \\
\hline 3 & 6.7488 & 24.6069 & 445.6983 \\
\hline
\end{tabular}

The second example studied is that of the plane frame, that is subjected to a lateral load of magnitude $\boldsymbol{F}$. The cross section of each beam and the material properties are the same as used in the previous example. The base of the structure is $b=4 \mathrm{~m}$ and each story is $h=4 \mathrm{~m}$ height. Each beam is divided in two elements of equal length. Finally, the horizontal displacement $u$ of the upper left node is measured. The displacement $u$ for different magnitudes of the load $\mathrm{F}$ is presented in Fig. (4).

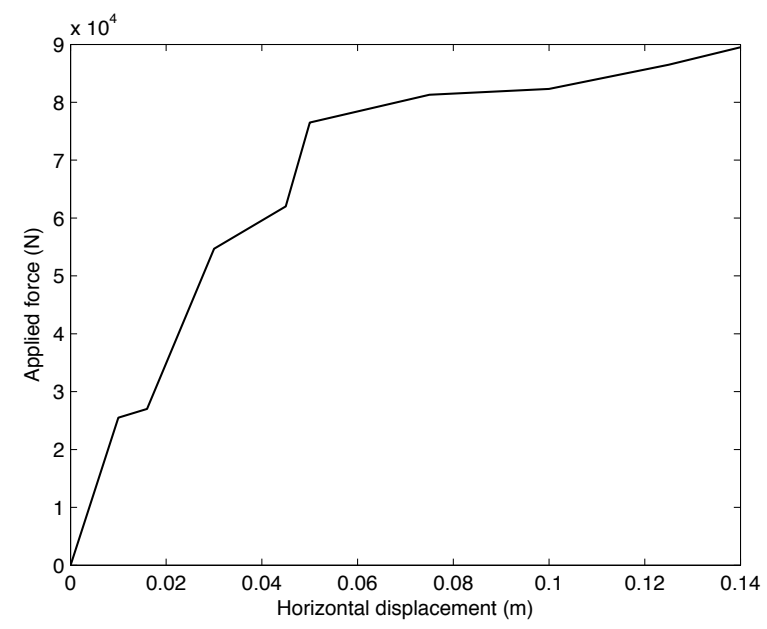

Fig. (4). Displacement $x$ applied force for the beam used in the second example.

\section{CONCLUSION}

This paper presents an approach for the reliability analysis of nonlinear reinforced concrete beams and frames, assuming maximum allowable displacements. The reliability analysis problem is solved using a FORM algorithm, and sensitivity analysis is carried out in an efficient manner (without using finite differences). The numerical results obtained here agree with those obtained from MC simulation. However, MC needs much more computational effort than FORM, mainly for structures with a high level of reliability.

It has been proved that every reliability index considering some finite allowable displacement is a lower bound for the reliability index when collapse is considered. This allows one to estimate the reliability index indirectly by solving a few reliability analysis problems considering maximum allowable displacements. The importance of this result is that reliability analysis considering collapse can lead to computational difficulties, due to the very nature of collapse.

In most cases the designer does not need to know the exact value of the reliability index of the structure, but just need to know if the reliability index is bigger than a minimum value. In these cases, the reliability index considering collapse can be estimated as described here. Besides, in many current practical applications constraints on maximum displacements must be enforced in order to guarantee an appropriate use of the construction.

\section{CONFLICT OF INTEREST}

The authors confirm that this article content has no conflict of interest.

\section{ACKNOWLEDGEMENTS}

Declared none.

\section{REFERENCES}

[1] S. P. Shah, J. S. Popovics, K. V. Subramaniam, and C. M. Aldea, "New directions in concrete health monitoring technology," Journal of Engineering Mechanics, vol. 126, no. 7, pp. 754-760, 2012.

[2] A. Lorenzi, E. G. Nesvijski, P. Sarkis, and J. Sarkis, "Infrastructure NDT monitoring using inspector-computer interface," In: Proceedings of ASNT Fall Conference and Quality Testing Show, Phoenix, Ariz, USA, 2009.

[3] E. G. Nesvijski, "Dry point contact transducers: design for new applications," The e-Journal of Nondestructive Testing, vol. 9, no. 9, 2003.

[4] C. L. Nogueira, "Analise ultra-sonica da distribuicao dos agregados no concreto atraves de wavelets," In: Proceedings of XXI Congresso Nacional de Ensaios Nao Destrutivos, Associacao Brasileira de Ensaios Nao Destrutivos, Salvador, Brazil, 2013, pp. 107-138.

[5] S. Shah, K. V. Subramaniam, and J. S. Popovics, "Use of nondestructive ultrasonic techniques for material assessment and inservice monitoring of concrete structures," In: Proceedings of the International Symposium on Nondestructive Testing Contribution to the Infrastructure Safety Systems in the $21^{\text {st }}$ Century, Universidade Federal de Santa Maria, Torres, Brazil, 2012, pp. 107-114.

[6] M. Schickert, M. Krause, and W. Muller, "Ultrasonic imaging of concrete elements using reconstruction by synthetic aperture focusing technique," Journal of Materials in Civil Engineering, vol. 15, no. 3, pp. 235-246, 2013.

[7] A. Mirmiran, and Y.Wei, "Damage Assessment of FRP-encased concrete using ultrasonic pulse velocity," Journal of Engineering Mechanics, vol. 127, no. 2, pp. 126-135, 2011.

[8] American Society for Nondestructive Testing (ASTM), "Standard test method for rebound hammer number of hardened concrete," Concrete and Aggregates, vol. 04, no. 02, pp. 400-402, 1999.

[9] M.D.A. Thomas, T. Bremner, and A.C.N. Scott, "Actual and modeled performance in a tidal zone", Concrete Int., vol. 33, no. 21, pp. 23-28, 2011. 